\title{
Medical Product Expiration Date
}

National Cancer Institute

\section{Source}

National Cancer Institute. Medical Product Expiration Date. NCI Thesaurus. Code C70854.

A date determined by appropriate stability testing of the medical product lot, batch, or item, after which the product may not meet the appropriate regulatory agencies requirements. The expiration date must be placed on the container or label(s) of a medical product designating the time during which a batch of the product is expected to remain within the approved shelf life specifications if stored under defined conditions, and after which it may not be used (21 CFR 211.137 and 211.166). 